\title{
Novel Case of 9p- Deletion in a Patient with Cardiac Pathology and a Review of the Literature
}

\author{
Koslow E ${ }^{1}$, Reeves $\mathrm{PT}^{1,2}$, Anchan, $\mathrm{J}^{2,3}$, Rohena $\mathrm{L}^{2,4}$ \\ ${ }^{1}$ San Antonio Uniformed Services Health Education Consortium, Department of Pediatrics, San Antonio, TX \\ ${ }^{2}$ F. Edward Hebert School of Medicine, Department of Pediatrics, Uniformed Services University of the Health Sciences, Department of Pediatrics, \\ Bethesda, MA \\ ${ }^{3}$ San Antonio Uniformed Services Health Education Consortium, Division of Neonatology, San Antonio, TX \\ ${ }^{4}$ San Antonio Uniformed Services Health Education Consortium, Division of Genetics, San Antonio, TX
}

${ }^{*}$ Correspondence Author: Elizabeth Koslow, 3551 Roger Brooke Drive, JBSA Ft Sam Houston, TX 78234; Fax (210) 916-9332, Office (210) 916-9928;

E-mail: Elizabeth.a.koslow.mil@mail.mil

Received: August 11, 2018; Accepted: August 21, 2018; Published: August 24, 2018;

\begin{abstract}
Our case focuses on a patient with a rare mutation, 9pter-9p22.2 interstitial deletion, associated with unique presentation and specific cardiac abnormalities which have not previously been associated with this condition. The subject was a term male infant born with an omphalocele which had been diagnosed prenatally. Upon delivery he was noted to have craniofacial and limb abnormalities, and found to be persistently hypoglycemic, requiring a significantly elevated glucose infusion rate. On echocardiogram an aortic coarctation was identified. He underwent cardiac repair, but ultimately developed severe pulmonary hypertension complicated by multiple episodes of cardiopulmonary arrest. To our knowledge there are only 26 previously identified patients with similar copy number variants on this region reprinted in the literature or identified in the Decipher database. This case report is not only able to help build knowledge which can be used to predict phenotypes, but it can also shed light on some of the more devastating characteristics possible with this condition. While this subject did have two of the most common symptoms involved, craniofacial and musculoskeletal, he also had cardiovascular abnormalities, which had only been identified in one other patient with 9pter-9p22.2 interstitial deletion. It was also a goal of this report to identify similarities and differences between 9pter-9p22.2 interstitial deletion with the much more well-known disease, $9 p$ minus syndrome. Of patients with $9 \mathrm{p}$ minus, craniofacial and musculoskeletal abnormalities are very common. Patients also frequently have visceral defects, intellectual disabilities, hypoglycemia, genital defects, and a small incidence of cardiovascular defects. More subjects are needed for further evaluation, but it appears that 9pter-9p22.2 interstitial deletion may be much more similar to the larger 9p minus syndrome than previously appreciated.
\end{abstract}

\section{Introduction/Background}

Current advancements in pediatric critical care as well as the evolution of human genomic investigations have revealed a wide range of clinical pathologies warranting further classification and, inturn, clinical correlations by providers. The DECIPHER database has focused on cataloguing specific mutations with correlated phenotypes to better characterize genetic abnormalities [1].

9p deletion syndrome is a pathologic genetic copy number variant initially identified in 1973 by Alfi and colleagues [2]. Since this time, more than 100 cases of $9 \mathrm{p}$ minus have been reported worldwide. The syndrome represents a heterogeneous condition, characterized upon initial presentation with craniofacial abnormalities (including trigonocephaly, midface hypoplasia, upslanting palpebral fissures, anteverted nostrils, depressed nasal bridge, long philtrum, and hypertelorism), short neck, increased inter-nipple distance, positional limb defects, nonketotic hypoglycemia, external genitalia anomalies, and cardiac abnormalities [3]. Follow up and longitudinal assessments demonstrate neurocognitive difficulties including low IQ (mean of
49), global developmental delays, hypotonia, and behavioral problems [4]. Visceral abnormalities including inguinal or umbilical hernias and omphaloceles have also been reported [5,6]. Swinkels et al. previously observed approximately $15 \%$ of patients with 9 p- syndrome had an omphalocele [7].

More specifically, 9pter-9p22.2 interstitial deletion, is a microdeletion syndrome which has demonstrated an emerging clinical significance and has currently been diagnosed in 26 patients across the globe. As shown in Table 1, these patients share many phenotypic similarities to 9pminus, but 9pter-9p22.2 interstitial deletion is subtyped with an even stronger association with craniofacial deformities and developmental delays [8].

With the apparent rise in the frequency of 9pminus diagnosis and concomitant presentations of the 9pter-9p22.2 interstitial deletion subtype, our report serves to catalogue and summarize the available data to provide initial diagnosing providers and follow up clinicians with a more robust clinical picture to best manage these complex patients. 
Table 1. Phenotypic abnormalities in known patients with 9pter-9p22.2 interstitial deletion [8].

\begin{tabular}{|l|c|c|}
\hline Phenotypic Abnormality & $\begin{array}{c}\text { Number with } \\
\text { abnormality (N = 26) }\end{array}$ & \% with abnormality \\
\hline $\begin{array}{l}\text { Head, ears, eyes, nose, } \\
\text { throat }\end{array}$ & 10 & 38.5 \\
\hline Developmental & 10 & 38.5 \\
\hline Musculoskeletal & 6 & 23.1 \\
\hline Endocrine & 3 & 11.5 \\
\hline Neurologic & 3 & 11.5 \\
\hline Skin & 1 & 3.8 \\
\hline Cardiovascular & 1 & 3.8 \\
\hline Gastrointestinal & 1 & 3.8 \\
\hline Genitourinary & 0 & 0.0 \\
\hline Pulmonology & 10 & 3.8 \\
\hline Fetal & 0.5 & 0.0 \\
\hline Other & 3 & \\
\hline Unknown & 3 & \\
\hline
\end{tabular}

\section{Clinical Report}

The subject of concern was a term male singleton born to a 25 year old G3P1 mother with insignificant past medical history. The pregnancy management involved a multi-disciplinary approach including maternal fetal medicine, neonatology, pediatric cardiology, and genetics due to concern for omphalocele with hepatic effect appreciated on prenatal anatomy ultrasound.

The child was delivered via scheduled cesarean section and received routine newborn resuscitation from the intensive care team with the use of a bowel bag. The initial transition period was complicated by hypoglycemia requiring a glucose intravenous infusion bolus in addition to an escalation of the glucose infusion rate to a maximum $9.9 \mathrm{mg} / \mathrm{kg} / \mathrm{min}$ at 24 hours of life.

The patient's remarkable findings on physical exam consisted of: frontal bossing with prominent metopic ridge, up-slanting palpebral fissures, posteriorly rotated ears; heart sounds auscultated with continuous machine-like murmur; $3 \mathrm{~cm}$ omphalocele; wide-spaced nipples; sandal gap deformity, talipes equinovares of the left foot.

Given the patient's constellation of clinical signs and symptoms, the neonatal team proceeded down the diagnostic pathway for Beckwith Wiedemann syndrome by obtaining an echocardiogram. The study revealed biventricular hypertrophy with normal function, a hypoplastic transverse arch with coarctation, and a PFO with left to right shunting. Following intubation in preparation for transfer, the patient was started on Prostaglandin $0.05 \mathrm{mcg} / \mathrm{kg} / \mathrm{min}$. Prior to transfer, and due to the presumptive clinical diagnosis of Beckwith Wiedemann, blood was obtained for genetic analysis. This investigation later revealed the etiology of the child's defects was 9pter-9p22.2 interstitial deletion.
After transfer to a higher level of care, the child underwent aortic arch repair. Preoperative echocardiogram demonstrated moderate to severe hypoplasia of the transverse and distal arch, multiple ventricular septal defects (perimembranous and muscular) with occasional bidirectional shunting, a stable atrial septal defect, as well as a bicuspid aortic valve. He ultimately required multiple surgeries including the previously stated aortic arch repair, hemidiaphragm plication, tracheostomy, gastrostomy-tube with Nissen fundoplication, and omphalocele repair. He spent several weeks in intensive care units during which multiple desaturation and code events occurred. Ultimately, at 5 months of life, palliative measures were put in place and shortly after the child died.

\section{Discussion}

The purpose of this report was to present the current knowledge known on 9pter-9p22.2 interstitial deletion, expand the available literature concerning the more well recognized $9 \mathrm{p}$ minus syndrome, and compare 9p minus syndrome to 9pter-9p22.2 interstitial deletion. This will ideally enable providers to better identify this subtype, as well as formulate appropriate treatment, surveillance, and prognostic plans.

The available cases of 9pter-9p22.2 interstitial deletion revealed craniofacial abnormalities (38.5\%), developmental delays (38.5\%) and musculoskeletal abnormalities (23\%) as the most common features typically identified. While our subject exhibited craniofacial and musculoskeletal abnormalities, he also demonstrated a less well recognized condition: congenital cardiac abnormalities. Prior to this report only one patient had been identified with cardiac dysfunction. While it is unclear specifically what cardiac abnormalities the previous patient had, our patient had significant cardiovascular compromise, including aortic coarctation, aortic hypoplasia, an atrial septal defect, ventricular septal defects and a bicuspid aortic valve. Due to such a small sample size, each additional patient with a specific feature, such as cardiac abnormalities has the potential to change the current associated risk related to that feature. Ultimately, subjects with the more common craniofacial and musculoskeletal abnormalities may warrant baseline echocardiograms and chest radiographs to evaluate for congenital cardiac defects. This may facilitate early recognition of deadly, congenital defects and hasten transfer, management and therapy at the appropriate facility.

\section{Conclusion}

Ideally, this report will aid clinicians in identifying and managing patients with 9pter-9p22.2 interstitial deletion. It is a significant copy number variant which, while sometimes fairly benign, has the potential to be devastating, and potentially fatal. Patients with confirmed or suspected 9pter-9p22.2 interstitial deletion would likely benefit from cardiovascular screening as well as potential transfer to a pediatric heart center.

Conflicts of Interest and Source of Funding: Salary support was provided for Drs. Koslow, Reeves, Anchan, and Rohena by the United States Department of Defense. The authors have no conflicts of interest. 
Disclaimer: This work was prepared as part of the official duties of Drs. Koslow, Reeves, Anchan, and Rohena who are employed by the United States Army and Air Force. The views expressed in this article are those of the authors and do not reflect the official policy or position of the United States Army, Air Force, Department of Defense, or the United States Government. Title 17 U.S.C. 105 provides that "Copyright protection under this title is not available for any work of the United States Government." Title 17 U.S.C. 101 defines a United States Government work as a work prepared by a military service member or employee of the United States Government as part of that person's official duties.

This study makes use of data generated by the DECIPHER community. A full list of centres who contributed to the generation of the data is available from http: //decipher.sanger.ac.uk and via email from decipher@sanger.ac.uk. Funding for the project was provided by Wellcome.

\section{Authors contributions:}

Elizabeth Koslow conceptualized the study, interpreted the data analysis, drafted the manuscript, and approved the final manuscript.

Patrick Reeves conceptualized the study, interpreted the data analysis, drafted the manuscript, and approved the final manuscript.

Joshua Anchan contributed to study design, developed the data analysis plan, interpreted the data, and revised the manuscript.

Luis Rohena contributed to study design, interpreted the data, and revised the manuscript.

All authors approved the final version of the manuscript.

\section{References}

1. Antonarakis, S. E. (2013). Human Gene Mutation in Inherited Disease: Molecular Mechanisms and Clinical Consequences. In D. Rimoin (Ed.), Emery and Rimoin's Essential Medical Genetics. Elsevier/AP.

2. Alfi OS. (1973) Delation of the short arm of chromosome\# 9 (46, 9P-): A new deletion syndrome. Ann Genet. 16: 17-22.

3. Boby, J., Karande, S., Lahiri, K., Jain, M., \& Kanade, S. (1994). 9p-Syndrome. Journal of Postgraduate Medicine. 40(1), 40-1.

4. Jones, K., Jones, M., \& Del Campo, M. (2013). Deletion 9P Syndrome (9P Monosome, 9P- Syndrome). In Smith's Recognizable Patterns of Human Malformation. Elsevier Health Sciences .

5. Spazzapan, P., Arnaud, E., Baujat, G., Nizon, M., Malan, V., Brunelle, F., \& Di Rocco , F. (2016). Clinical and neuroradiological features of the 9p deletion syndrome. Child's Nervous System, 32(2), 327-335. [Crossref]

6. Hou, Q.-F., Wu, D., Chu, Y., \& Liao, S.-X. (2016). Clinical findings and molecular cytogenetic study of de novo pure chromosome 9p deletion: Pre- and postnatal diagnosis. Taiwanese Journal of Obstetrics and Gynecology, 55(6), 867-870. [Crossref]

7. Swinkels, M., Simons, A., Smeets, D., Vissers, L., Veltman, J., Pfundt, R., . van Ravenswaaii-Arts, C. (2008). Clinical and cytogenetic characterization of 13 Dutch patients with deletion 9p syndrome: Delineation of the critical region for a consensus phenotype. America Journal of Medical Genetics A, 146A(11), 1430-8. [Crossref]

8. Firth, H., Richards, S., Bevan , A., Clayton, S., Corphas, M., Rajan, D., . . Carter, N. (2009). DECIPHER: database of chromosomal imbalance and phenotype in humans using ensemble resources. The American Journal of Human Genetics , 84(4), 524-33. [Crossref]

\section{Citation:}

Koslow E, Reeves PT, Anchan, J, Rohena L (2018) Novel Case of 9p- Deletion in a Patient with Cardiac Pathology and a Review of the Literature. J Clin Res Med Volume 1(2): 1-3 